\title{
Ambulatory anesthesia in plastic surgery: opportunities and challenges
}

This article was published in the following Dove Press journal:

Ambulatory Anesthesia

I October 2015

Number of times this article has been viewed

\section{Alexander R Facque \\ Peter J Taub}

Division of Plastic and Reconstructive Surgery, Department of Surgery, Mount Sinai Medical Center, New York, NY, USA
Correspondence: Peter J Taub Division of Plastic and Reconstructive Surgery, Department of Surgery, Mount Sinai Medical Center, 5 East 98th Street, 14th Floor, Suite B, Box 1259, New York, NY 10029, USA

Tel + I 2 I $224 \mid 5873$

Fax + I 2125342654

Email peter.taub@mountsinai.org
Abstract: In 2013, there were 17 million procedures performed by plastic and reconstructive surgeons in the United States in the private office or ambulatory "surgicenter" setting, as well as additional operations performed in hospitals on an outpatient basis. As interest in performing increasingly complex surgical procedures on an outpatient basis continues to grow, the surgeon and anesthesiologist alike must be prepared to offer safe and reliable anesthesia and analgesia in the ambulatory setting. Surgeons must be aware of the possible techniques that will be employed in their surgeries in order to anticipate and prepare patients for possible postoperative side effects, and anesthesiologists must be prepared to offer such techniques in order to ensure a relatively rapid return to normal activity despite potentially having undergone major surgery. The following is a review of the specific considerations that should be given to ambulatory plastic surgery patients with comments on recent developments in the techniques used to safely administer agreeable and effective anesthesia.

Keywords: ambulatory surgery, cosmetic anesthesia, outpatient, ambulatory anesthesia

\section{Introduction}

According to the statistics presented by the American Society of Plastic Surgery, more than 20 million cosmetic and reconstructive procedures were performed in 2013 in the United States. Of these, approximately 13 million were performed in an office setting and nearly 4 million were performed in ambulatory surgery centers (Figure 1), which is an increase of 3\%-4\% compared with the statistics released for $2012 .{ }^{1}$ This interest in performing an increasing number of elective operations on an ambulatory basis is present across all surgical specialties as reflected in the presence and increase in the number of freestanding surgery centers. ${ }^{2}$ As the demand for non-hospital-based procedures increases each year, developing reliable anesthesia techniques that maximize patient safety while preventing discomfort and prolonged post-procedure aftereffects is of paramount importance in the plastic surgeon's ability to offer these procedures in an ambulatory setting and avoid costly and inconvenient inpatient hospitalizations.

As important as the advances in developing less invasive surgical techniques, the availability of rapid-onset, rapid-emergence, short-acting anesthetic and analgesic agents is of paramount importance in offering plastic surgery on an elective basis. Plastic surgery is unique in its large percentage of elective cases and the associated necessity of providing a highly comfortable patient experience. In most cases, the majority of patients are self-paying, without the assistance of insurance, and again, the overall experience, including their interactions with staff in the facility to the anesthetic agents used, is under extreme scrutiny. The plastic surgeon and 


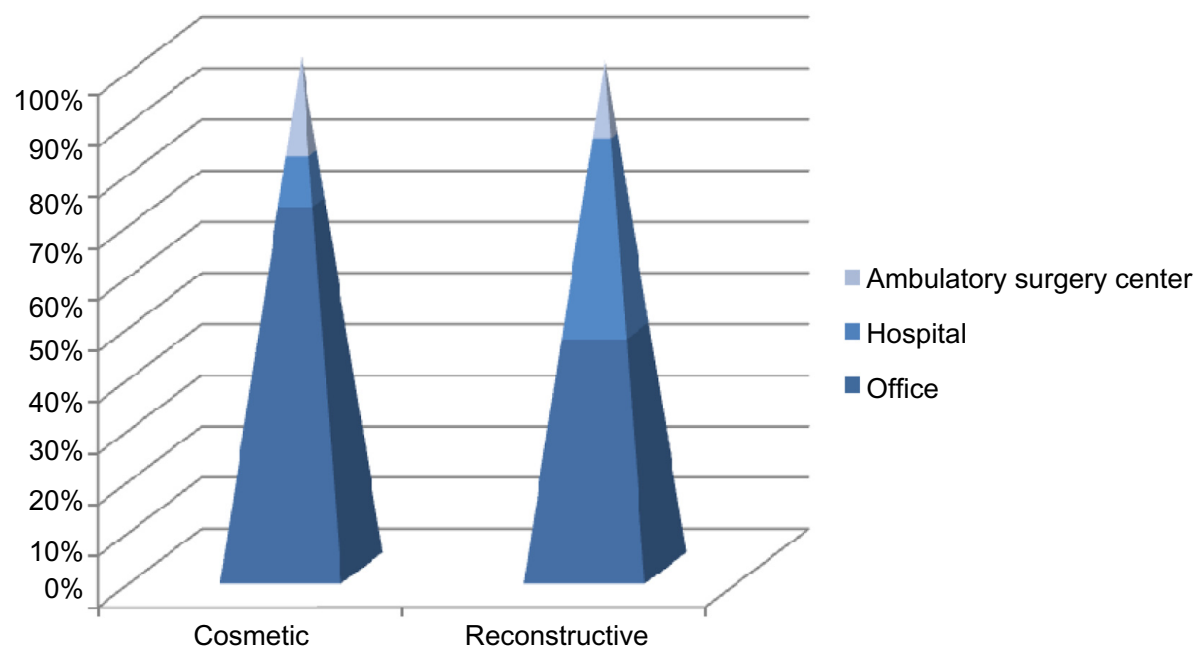

Figure I Plastic surgery procedures in 2013 categorized by location.

Notes: Of the 20.8 million procedures reported in 20I3, 15.1 million were cosmetic, including minimally invasive cosmetic procedures, and the remaining 5.7 million reconstructive. In total, 13.4 million were office-based, 3.6 million were performed in the hospital setting, and 3.7 million in free-standing ambulatory surgery facilities. Data from the 2013 annual statistics presented by the American Society of Plastic Surgeons.

anesthesiologist must work in tandem, each having an excellent understanding of the other's contribution to the overall experience. The surgeon will be responsible for preliminarily explaining and managing the patient's expectations of the perioperative period and must have an understanding of the anesthetic technique in order to properly address this. Likewise, the anesthesiologist will need to understand the surgical procedure, in order to choose the most appropriate technique with attention to pre- and postoperative symptom management. With both physicians working together, the goals of maximized patient safety and an overall pleasant experience can be achieved.

In simple terms, the goal and challenge of providing ambulatory anesthesia in relation to the plastic surgeon and the patient is to provide safe and reliable anesthesia and analgesia for potentially major operations while minimizing postoperative effects and complications of the anesthetics themselves. This will allow the patient to have the ability to safely return home in the immediate postoperative period and a relatively rapid return to normal function.

\section{Risk assessment strategies}

Prior to any consideration of the various formulations and intricacies of the delivered anesthetic technique, careful attention to patient selection is of paramount importance. As patient safety is the ultimate goal, choosing which patients may safely undergo a potentially major operation and have the ability to return home the same day is just as important as any other component of the procedure. Patients should undergo a rigorous screening process, and some will benefit from medical optimization prior to receiving any type of anesthesia. However, the question of who should be medically optimized and to what extent is at the forefront of deciding who will be allowed to undergo an elective cosmetic or reconstructive procedure. In an attempt to assess the risk associated with anesthesia and surgery related to a patient's premorbid conditions, in 1941, at the request of the American Society of Anesthesiologists (ASA), Saklad developed the ASA Physical Status score (or ASA). This classification scheme has become almost universally present in the surgical setting and is a widely recognized risk stratification tool shown to correlate well with postoperative outcomes. Notably, in its development, neither the type of surgery nor the technique of anesthesia was considered. The system gives a subjective and relative risk based on the patient's preoperative medical history, and studies show that the ASA score correlates well with unplanned admissions, perioperative complications, and surgical risk.

In this classification system, each patient can be assigned a position on a continuum based on his or her physical condition and the presence of systemic disease ranging from completely health (Class 1) to organ donor (Class 6) (Figure 2). ${ }^{3}$ In general, patients classified as ASA 1 or 2 will not need to undergo further evaluation prior to surgery, whereas it is widely practiced that patients belonging to ASA class 3 would likely benefit from additional assessment. However, a large prospective study demonstrated that $24 \%$ of ambulatory surgery patients were ASA class 3 and that these patients had the same low incidence of morbidity as ASA class 1 and class 2 patients in this setting. ${ }^{4}$ 


\begin{tabular}{|c|c|}
\hline ASA classification & Description \\
\hline 1 & A normal, healthy patient \\
\hline 2 & A patient with mild systemic disease \\
\hline 3 & A patient with severe, systemic disease \\
\hline 4 & A patient with severe, systemic disease that is a constant threat to life \\
\hline 5 & $\begin{array}{l}\text { A moribund patient who is not expected to survive without the } \\
\text { operation }\end{array}$ \\
\hline 6 & $\begin{array}{l}\text { A declared brain-dead patient whose organs are being removed for } \\
\text { donation }\end{array}$ \\
\hline
\end{tabular}

Figure 2 ASA Physical Status score.

Notes: Most patient electing to undergo cosmetic surgery will be classified as ASA I or 2, and will not need to undergo further evaluation prior to surgery; however, with reconstructive plastic surgery, which may also be offered on an ambulatory basis, patients may be more often classified as ASA 3 and would likely benefit from additional assessment. $^{3}$

Abbreviation: ASA, American Society of Anesthesiologists.

A separate classification system, specific to cardiac risk assessment for noncardiac procedures, was published by the ACC/AHA in 2007 and recently revised in 2009. In this system, three elements contribute to assess the perioperative cardiac risk: patient-specific clinical variables, exercise capacity, and surgery-specific risks. Surgical procedures are stratified into high, intermediate, and low risk, with ambulatory surgery generally considered low risk with an accompanying risk of cardiac events at less than $1 \%$. Patient-specific variables are split into active cardiac conditions, such as unstable coronary syndrome or decompensated heart failure, and clinical risk factors, such as ischemic heart disease, compensated congestive heart failure, cerebrovascular disease, treatment with insulin, and elevated creatinine. The presence of these factors correlates well with perioperative cardiac risk and, in fact, the presence of two or three or more variables is accompanied by a $7 \%$ and $11 \%$ risk of cardiac complications, respectively. ${ }^{5}$ In the ambulatory surgery setting - again considered low risk in the guideline's risk stratification of the surgical procedure - patients with at least one clinical risk factor warrant further optimization and evaluation prior to scheduling. Patients with active cardiac conditions are not candidates for elective surgery and should be encouraged to present to a primary care physician or cardiologist for evaluation and treatment.
Interestingly, among these risk assessment schemes, age alone is not considered an independent risk factor when considering suitability for outpatient surgery, and, in fact, studies demonstrate that elderly patients experience less postoperative pain, dizziness, and emetic symptoms than their younger counterparts. ${ }^{6}$ Nevertheless, due to multiple comorbidities, elderly outpatients may experience a higher incidence of perioperative cardiovascular events, and recovery of fine motor skills may be slowed with increasing age. ${ }^{7}$ Due to a greater need for supervision post-procedure, social factors including lack of appropriate transportation, a responsible escort, or the presence of a caretaker at home take precedence when offering same-day surgery to those of advanced age, and elderly patients may subsequently be found less suitable to undergo outpatient surgery for these factors as well.

As plastic surgery is a field offering services to those of all ages, at the other end of life, ex-premature infants recovering from minor procedures under general anesthesia are noted to have an increased incidence of postoperative apnea, which has been thought to extend to the 60th week of life. ${ }^{8,9}$ Subsequently, such infants will require a high degree of postoperative monitoring in an inpatient setting until the surgeon and the anesthesiologist are comfortable that this risk has abated to an acceptable level. 


\section{Preoperative evaluation and risk assessment}

With the assistance of largely subjective risk assessment tools, the physician's greatest assistance in identifying any conditions that could endanger a patient's ability to withstand the physiologic stress of surgery is the history and physical examination. An evaluation of past medical problems and their degree of optimization, past surgical and anesthetic history, specifically with relation to complications, current medications (including herbal remedies), and the presence of allergies alone identifies $86 \%$ of patient diagnoses (an additional $6 \%$ were identified with the physical exam, and a final $8 \%$ were determined with additional laboratory testing). ${ }^{10}$

\section{Lab pretesting}

The prevalence of unrecognized disease that influences surgical risk is low in healthy individuals. Nevertheless, clinicians often perform laboratory tests in this group of patients out of habit and medicolegal concern, with little benefit and a high incidence of false-positive results. Normal test values are usually arbitrarily defined as those occurring within two standard deviations from the mean, thereby ensuring that $5 \%$ of healthy individuals who have a single screening test will have an abnormal result. As more tests are ordered, the likelihood of a false-positive test result increases; a screening panel containing 20 independent tests in a patient with no disease will yield at least one abnormal result $64 \%$ of the time. ${ }^{11}$ Thus, the predictive value of abnormal test results is low in healthy patients with a low prevalence of disease. Aside from possibly causing patient alarm, the additional testing prompted by false-positive screening tests leads to unnecessary costs, risks, and a potential delay of surgery. In addition, clinicians often fail to act upon abnormal test results from routine preoperative testing, thereby actually creating an additional medicolegal risk. Subsequently, for ambulatory surgery procedures, deemed low risk, routine testing is not recommended, and, in fact, a practice advisory from the ASA and a safety guideline from the Association of Anaesthetists of Great Britain and Ireland recommend against routine preoperative laboratory testing in the absence of clinical indications. ${ }^{12,13}$

Directed preoperative testing is recommended when clinically indicated on a per patient and procedure basis. Selective testing is appropriate in specific circumstances, including patients with known underlying diseases or risk factors where the result would affect operative management or confer increased risk. Specific laboratory studies commonly ordered for preoperative evaluation include a complete blood count for patients at the extremes of age or with history of hematologic or liver diseases. A complete blood count is also of value in menstruating women patients depending on the degree of anticipated blood loss. Ordering an electrolyte panel and renal function assay for those patients with endocrine or renal disorders, and blood glucose for the obese may also be indicated. As diabetes is a disease with high prevalence in the overweight and obese populations, it does not fit the examples given above and screening is viewed as appropriate in those circumstances. Additional studies to consider include liver function studies, hemostasis evaluation, and urinalysis. Again, as there is a relatively high incidence of unknown pregnancy, this is an encouraged and often required screening laboratory test for women.

\section{Preoperative electrocardiogram}

High-risk patients or patients undergoing vascular or other high-risk procedure are strongly recommended to have at least a preoperative electrocardiogram (EKG). In relation to the relatively lower risk ambulatory procedures, in patients that have already been predetermined to be of lower risk, routine EKG has limited utility. Subsequently, the ACC/AHA guidelines released in 2007 states that an EKG is not indicated in asymptomatic patients undergoing low-risk surgery. ${ }^{5}$ Patients undergoing intermediate risk surgery with least one clinical risk factor are recommended to have a preoperative EKG performed.

\section{Chest radiographs and pulmonary function testing}

There is little evidence to support the use of a preoperative chest radiograph regardless of age unless there is known or suspected cardiopulmonary disease from the history or physical examination. In a meta-analysis of 21 studies of routine chest radiography, among a total of 14,390 routine chest X-rays, there were 1,444 abnormal studies. Only 140 abnormal findings were unexpected, and only $14(0.1 \%)$ of all routine chest X-rays influenced management. ${ }^{14}$ Likewise, pulmonary function testing is rarely indicated but should be considered in patients with a history of lung resection, radiation, or decreased functional capacity.

PerASA task force on preanesthesia evaluation, tests within 6 months are generally acceptable if no recent changes are noted. Scheduling an appointment for preoperative assessment 1-2 weeks before surgery was found to reduce preoperative anxiety when compared with assessment on the evening before surgery. ${ }^{15}$ This visit can be used for patient education, financial review, explanation of surgery-day procedures, and paperwork 
review, and may be useful to avoid cancellations, no shows, and substandard perioperative care.

\section{Obstructive sleep apnea}

Obstructive sleep apnea (OSA) is a very prevalent and important condition to consider in the evaluation of the ambulatory surgery patient, as it has associations with significant perioperative complications, and, once identified, relatively simple alterations in anesthetic technique may be employed to greatly increase patient safety. Mild OSA, characterized by $5-15$ breathing pauses per hour during sleep, is found in one in four men and one in ten women, classifying OSA as the most common diagnosis of sleep-disordered breathing. ${ }^{16}$ Furthermore, recent studies indicate that this diagnosis is becoming even more common, paralleling the obesity epidemic we now see worldwide. Finally, OSA has also been found to increase surgical risk, both in the intraoperative and postoperative periods, making screening for OSA an increasingly important part of the evaluation of an ambulatory surgery candidate.

A 2012 meta-analysis of 13 studies showed that OSA increased the odds of the postoperative and intraoperative complications by factors of 2-4 depending on the complication. ${ }^{17}$ These complications were most frequently respiratory, including OSA exacerbation, desaturation, acute respiratory failure, and reintubation. Increased cardiac events, myocardial infarct/ischemia, arrhythmia, cardiac arrest, and increased length of hospital stay were also reported. Although not demonstrated in a recent retrospective analysis, it is generally believed that the severity of OSA (graded by number of apneic events during sleep) is correlated with these worse outcomes. $^{18}$

Due to the high prevalence of the disease, screening is relatively straightforward and can be accomplished effectively with a short questionnaire. Some of the most common include the STOP BANG, ${ }^{19}$ sleep apnea clinical score,${ }^{20}$ and the Berlin ${ }^{21}$ questionnaires, each reported in the literature as having sufficiently high sensitivity. These questionnaires assess various combinations of daytime sleepiness, neck circumference, witnessed events, and comorbid conditions such as obesity and hypertension (HTN). Consequently, we recommend that all patients be screened for OSA; however, the most clinical suspicion is directed toward the obese and those with conditions in which concomitant OSA is prevalent such as HTN and type 2 diabetes mellitus. OSA is also seen at higher rates in those with congestive heart failure and stroke, although these conditions themselves would warrant further evaluation and may preclude the patient from ambulatory surgery. Following screening those with a positive questionnaire or suspicious of the disease can then be directed toward definitive diagnosis - the gold standard being a sleep polysomnogram - and management by a specialist. In patients with established OSA, it is important to assess the severity, adequacy, and compliance of current treatment, as well as to document current treatment.

Ultimately, patients with controlled low/moderateseverity disease or even suspected OSA may proceed to low-risk procedures provided the postoperative narcotic requirement is not too high. A 2012 consensus statement reported that low-risk ambulatory surgery can be performed in patients with known or presumed OSA if these patients have well-controlled comorbidities and an ability to comply with recommendations for postoperative positive airway pressure therapy. ${ }^{21}$ Patients with OSA should continue their current treatment up to surgery, be prepared to continue afterward as soon as feasible, and should be instructed to bring their equipment on the day of surgery, should the need arise postoperatively for its use. Patients with severe or unoptimized OSA should seek treatment prior to scheduling surgery, and surgery should be delayed until the condition is considered controlled and the patient is compliant with treatment. Careful attention should be made in the evaluation of patients with OSA within the realm of plastic surgery, as many procedures are considered ambulatory and low risk yet may also come with a substantial postoperative narcotic requirement. In these patients, performing surgery in a setting with ability for more focused and longer postoperative monitoring and arrangements for conversion to a hospital stay if needed may be necessary.

\section{Smoking cessation}

All patients should be encouraged to quit smoking, for general health concerns; however, preoperatively this gains even more importance. Smoking cessation has been shown repeatedly in both case-control and cohort studies to reduce postoperative morbidity and mortality. In an extraction of 157 datasets, smoking prior to surgery increased the risk of wound complications, infection, pulmonary complications, neurological events, and postoperative admissions to the intensive care unit. ${ }^{23}$ Furthermore, in relation to the timing of cessation, a 2012 meta-analysis demonstrated that smokers who quit smoking 8 and 4 weeks prior to surgery had significantly lower risks of respiratory complications ( $R R=0.77$ and 0.53 , respectively). Those who quit 3-4 weeks before had lower wound healing complications $(\mathrm{RR}=0.69)$, and smokers who quit less than 4 weeks prior had similar 
rates of respiratory complications as current smokers. ${ }^{24}$ In conclusion, smokers should be encouraged to quit as far in advance of their surgical procedure as possible, with the greatest benefit being found with cessation greater than 4 weeks before operation.

\section{NPO guidelines}

Currently the ASA guidelines for preoperative fasting include abstaining from clear liquids 2 hours prior to surgery, avoiding light meals for 6 hours, and 8 hours for a meal with fried of fatty foods. Subsequently, for simplicity and, hence, greatest compliance, most practitioners suggest an all-encompassing fasting period of 8 hours, or nothing by mouth after midnight before surgery. However, this practice leaves patients dehydrated, potentially hypoglycemic, and possibility in a catabolic state at the time of surgery. Additionally, to be in physical and mental discomfort that comes with being in such a hungry and thirsty state is also distressing to patients. It has been shown that adequate hydration (eg, allowing oral intake of clear liquids up to 2-3 hours before surgery and/or IV hydration before induction of anesthesia) is associated with a decreased incidence of postoperative side effects, including pain, dizziness, drowsiness, thirst, and nausea. ${ }^{25,26}$ Due to short half-life of clear fluids in the stomach (10-20 minutes), residual gastric volume after 2 hours is actually less in patients ingesting small amounts of clear fluids than in fasted patients. ${ }^{27}$ Therefore, as prolonged fasting does not guarantee an empty stomach at the time of induction, several investigators have even questioned the value of relatively short 4- to 5-hour fasts before elective surgery. ${ }^{28-31}$ In these studies, drinking $150 \mathrm{~mL}$ of water 2 hours before surgery significantly decreased the severity of thirst without increasing gastric volume in fasted outpatients. ${ }^{32}$ Ingestion of $150 \mathrm{~mL}$ of either coffee or orange juice $2-3$ hours before induction of anesthesia had no significant effect on residual gastric volume or $\mathrm{pH}$ even in obese adults. ${ }^{32,33}$ Ultimately, further research will need to be directed toward clinical outcomes regarding updated NPO recommendations, but importantly for patient's currently undergoing surgery, the standard of care and ASA guidelines continue to reflect the 8-hour NPO dictum.

\section{Care in the immediate preoperative period}

Understandably, it is very common for patients to feel anxiety and apprehension before surgery, and in order to address this, a number of pharmacologic and nonpharmacologic measures have been described. In addition to urging patients to get plenty of sleep the night before the procedure, some practitioners offer a medication that provides somnolence, such as a benzodiazepine, which will also offer additional anxiolysis. In fact, many plastic surgeons treat preoperative anxiety prophylactically. Anxiolytic medications such as alprazolam or lorazepam can be prescribed to patients 1-3 days prior to the procedure in doses of $0.25-0.50 \mathrm{mg}$ three times daily and 2-4 mg twice per day, respectively. Additionally, clonidine, an alpha-2 agonist, given in doses of $0.1-0.2 \mathrm{mg}$ orally in the morning of surgery has been shown to be dually beneficial by both decreasing blood pressure and providing a degree of sedation. Informed consent for the surgical procedure should be obtained in advance of the surgery, and many anesthesiologists do not feel that a small dose of a preoperative anxiolytic will interfere with the patient's ability to sign consent for anesthesia. ${ }^{32}$ Ultimately, procedures performed on a well-relaxed patient will more than likely be much smoother than those performed on an irritable and anxious patient.

Nonpharmacologic methods to reduce anxiety are varied. In 1989 it was demonstrated that the preoperative visit with the anesthesiologist was more effective than pharmacologic interventions in reducing preoperative anxiety. ${ }^{33}$ Postoperative pain has been shown to be reduced with preoperative educational programs that could be included in the same preoperative visit with the anesthesiologist. Investigators have found that anxiety was significantly reduced when this visit was 1-2 weeks prior to surgery compared with an assessment the evening of surgery. ${ }^{2,5}$

\section{Preinduction}

Once in the operating room, continuous EKG, cycling blood pressure cuff, oxygen saturation monitor, end tidal carbon dioxide monitor, and temperature probe are placed on the patient per ASA guidelines. A neuromuscular monitor can be placed for cases involving paralysis, and placement of a cerebral activity monitor may be considered as well. Compression boots should be placed for deep venous thrombosis prophylaxis. A bladder catheter should be used in cases greater than 3 hours or in circumstances with the potential for large-volume fluid resuscitation or with bladder decompression, such as an abdominoplasty with liposuction.

\section{Cerebral monitoring}

While a patient's vital signs remains the most useful method for determining the depth of anesthesia during surgery, the value of cerebral monitoring as an adjuvant to monitoring autonomic responses has increased in popularity and 
prevalence. One such device is the bispectral index (BIS). From Miller's Anesthesia, "the BIS is an empirically derived scale that was proposed by Aspect Medical Systems (later purchased by Covidien [Boulder, CO, USA]) in 1994, as a novel way to monitor level of consciousness among patients receiving general anesthesia and sedation". ${ }^{36}$ The value reflected in the BIS falls between 0 and 100, with 0 being without electrical activity on EEG and 100 registering as completely awake. Typically, values of the properly anesthetized patient range between 40 and 60. Several studies of BIS monitoring have reported that the use of cerebral monitoring can improve early recovery after general anesthesia in the ambulatory setting because of its ability to titrate anesthetics and thereby minimize both "overdosing" and "underdosing" with both intravenous (eg, propofol) and inhaled (eg, sevoflurane and desflurane) anesthetic drugs during the maintenance period..$^{37}$ As a result of the anesthetic sparing effects of the use of cerebral monitors, additional studies have demonstrated a 30- to 90-minute reduction in the time to discharge home after ambulatory surgery. ${ }^{38}$

\section{Patient positioning}

Positioning is of special interest to the plastic surgeon, as many procedures carry a high risk of patient injury due to their potentially long duration, complicated patient positions, and possible requirement for intraoperative position changes. For instance, medial thigh lifts are frequently performed in lithotomy position, leading to increased risk of deep venous thrombosis or femoral nerve neuropraxia. A belt lipectomy will involve intraoperative repositioning with increased risk of trauma during the move. In breast surgery, many surgeons will flex a patient's torso during the operation, potentially repeatedly, and care should be taken to avoid dislodgement of the endotracheal tube as well transient hypotension in the flexed position. Known areas of possible prolonged nerve pressure should always be padded and, in all cases, areas of the skin exposed to firm pressure should be padded to prevent pressure related skin damage and ulceration.

\section{Temperature}

Patients receiving general anesthesia are at an increased susceptibility to hypothermia in the operating room. This occurs due to derangement in thermoregulation, resulting from the inability to vasoconstrict and shiver, combined with the lower ambient temperature of the operating room and necessary exposure of the patient. Large randomized trials have proved that even mild hypothermia (ie, $1.5^{\circ} \mathrm{C}-2.0^{\circ} \mathrm{C}$ ) leads to adverse outcomes, including a threefold increase in morbid myocardial outcomes, a threefold increase in risk for wound infection, coagulopathy, and need for allogeneic transfusion, prolonged recovery, and prolonged hospitalization. ${ }^{39-41}$ Bleeding diathesis due to cold-induced platelet dysfunction, decreased wound healing possibly related to triggered thermoregulatory vasoconstriction, and increased incidence of infection (thought to be due to a decrease in the enzymatic activity of the patient's host immune responses) have also been described. ${ }^{42,43}$ Thus, the ambient temperature of the operating room should be kept at a level that minimizes body heat loss, and a warming blanket should be used for longer procedures. While hypothermia is probably not a likely occurrence in short cases, a warming blanket is relatively inexpensive compared to other adjunctive devices with little risk of morbidity to the patient.

\section{Induction and general anesthesia}

Induction of anesthesia is usually obtained with a rapidacting intravenous anesthetic. Propofol has replaced barbiturates due to its quick onset, fast recovery, and decreased potential for postoperative side effects. ${ }^{44}$ The most popular technique for maintenance of anesthesia is a combination of a volatile anesthetic with or without nitrous oxide (NO). The low solubility of newer volatile agents and $\mathrm{NO}$ contribute to a more rapid recovery from anesthesia at the conclusion of the procedure. Importantly, the emetogenic potential of NO in this case is outweighed by its anesthetic and analgesic sparing effects. A separate method of maintenance of anesthesia is total intravenous anesthesia, which utilizes a combination of propofol and a narcotic agent for analgesia, such as remifentanil or alfentanil. Studies suggest that total intravenous anesthesia is as effective as spinal anesthesia with the added benefit of decreased post-anesthesia care unit (PACU) recovery time prior to home readiness. ${ }^{45,46}$ Despite advantages gained by in local and regional therapy, general anesthesia remains popular and is associated with a higher incident of post-anesthetic side effects and prolonged PACU stay, as well as an increase in need for ancillary equipment such as padding, heated airway humidifiers, and forced warmers for patient comfort.

For airway protection, a breathing tube is often placed when administering general anesthesia. To alleviate the common complaint of sore throat postoperatively, in 1983 the laryngeal mask airway was introduced as a minimally invasive airway alternate to the endotracheal tube in securing an airway. The laryngeal mask airway has the advantage of being easily positioned without direct visualization or neuromuscular blockade. Patients ventilate spontaneously 
throughout the procedure, resulting in a decreased time to wake up at the conclusion of the case. ${ }^{47}$

\section{Intraoperative management of patients with OSA}

Patients with OSA are considered more susceptible to the respiratory depressant action of many drugs, with increased propensity toward upper airway obstruction (especially in the supine position), and a potentially difficult airway. As such, a general principle is to use an anesthetic technique that minimizes the use of respiratory depressants whose effects will continue into the postoperative period, avoid supine positioning as much as possible, and always have available assistance and equipment should the need to establish a difficult airway arise. In sedated patients, ventilation and oxygenation should be monitored, and the patient may need to be provided with continuous positive airway pressure via their home device or an oral airway to ensure adequate oxygenation and subsequently should be instructed in a preoperative visit to make this equipment available on the day of surgery. ${ }^{36}$

Specific recommendations by the ASA, in a guideline consensus on patients with OSA from 2014, include overall techniques to reduce sedative agents by choosing shortacting agents, those with decreased respiratory depressant potential, using a minimal amount of medication to achieve the desired effect (eg, by considering an infusion of sedative over a large dose bolus), and explore the possibility of providing regional anesthesia techniques whenever possible. As difficulties in laryngoscopy and intubation are common in those with OSA, preparing both routine and emergency equipment and planning for the possibility of advanced airway techniques or awake intubation should be considered. Therefore, the guideline consensus also recommends ensuring the presence of skilled assistance and to consider positioning with a ramp technique or to employ both nasopharyngeal and oropharyngeal airways to optimize the sniffing position. They further recommend that extubation should take place with the head up, with airway reflexes intact, neuromuscular blocking agents reversed, and with adequate ventilation. ${ }^{36}$

\section{Monitored anesthesia care}

The combination of local anesthesia and/or peripheral nerve blocks with intravenous sedative and analgesic drugs is commonly referred to as monitored anesthesia care (MAC) and has become extremely popular in the ambulatory setting. ${ }^{48}$ Compared to general anesthesia, MAC is associated with decreased total operating room time, decreased time to wake up and orientation, decreased time to home readiness, decreased postoperative nausea and vomiting (PONV), decreased postoperative pain, and increased patient satisfaction. ${ }^{49}$ Importantly, vigilant monitoring is required, as patients may rapidly progress from a "light" level of sedation to "deep" sedation (or unconsciousness) and, thus, may be at risk for airway obstruction, oxygen desaturation, and even aspiration. Although MAC is associated with the lowest incidence of postoperative side effects, there are other MAC-specific risks, such as the possibility of transient nerve palsy when peripheral nerve block techniques are used, which should receive consideration..$^{50}$

Despite the advantages provided by administering MAC, general anesthesia remains popular among providers and patients, partially related to the fact that many patients simply prefer to "be asleep during the operation." Furthermore, in plastic surgery, where procedures are performed on an elective basis and are subsequently considered low risk, the procedures themselves may be quite extensive (ie, belt lipectomy, large reduction mammaplasty) such that general anesthesia will be required for patient safety. Necessarily, a discussion between the surgeon, anesthesiologist, and patient will best determine which method of anesthesia is appropriate to render the patient free of discomfort while also allowing for minimal postoperative side effects and ultimately a safe and comfortable discharge home following the surgery.

\section{Local/regional}

Compared to both MAC and general anesthesia, procedures on the hand and face that can be performed with local or regional anesthesia have the benefit of faster recovery, minimal side effects like PONV, or respiratory complications, and offer a cost advantage through decreased need for nursing care postoperatively. Peripheral nerve blocks are a valuable supplement to both general anesthesia and MAC techniques. Safe and reliable blocks that offer complete analgesia to the face and hand have been well described in plastic surgery literature. For the face specifically, potential blocks to the infraorbital, mental, supraorbital, dorsal nasal, zygomaticotemporal and zygomaticofacial nerves may be performed in various combinations to allow larger procedures performed under local anesthesia. ${ }^{51}$ Techniques are also described to make it possible for patients to feel only the initial injection. These include using buffered local anesthetic, warming the solution, distracting the patient, using smaller needles, application of topical numbing agents, slow 
injection speed, and, importantly, gaining patient feedback of the pain of anesthetic injection to improve performer skill. ${ }^{52}$ Furthermore, liposomal bupivacaine can provide up to 72 hours of local pain relief and has proven safety and efficacy. ${ }^{53} \mathrm{~A}$ study of liposomal bupivacaine injection performed as a regional block in abdominoplasty with rectus plication found that patients experienced reduced postoperative pain, required less postoperative narcotic medication, and resumed both earlier ambulation and normal activity compared to controls. ${ }^{54}$

\section{Spinal/epidural anesthesia}

Spinal or epidural anesthesia may be of benefit in the cases of abdominal, breast, and lower extremity procedures. Spinal anesthesia is considered more reliable with a faster onset, whereas the epidural is more technically difficult but has the ability to be continuous with catheter placement at the conclusion of the procedure. A combination technique utilizing both spinal and epidural techniques allows fast onset with the flexibility of continuous epidural anesthesia. The small initial dose of intrathecal drug results in a more rapid onset, reduced side effects, and faster recovery from the sensorimotor blockade. If necessary, the epidural catheter could be used to extend the block beyond the duration of the spinal anesthetic. ${ }^{55,56}$

\section{Intravenous regional anesthesia}

For short ( $<60$ minutes) superficial surgery involving one extremity, IV regional anesthesia (Bier's block) is a simple and reliable technique that has been found to be more cost effective than general anesthesia for surgery involving the hand, and can be safely performed by both the surgeon and anesthesiologist with good results. ${ }^{57}$ In this technique, a double tourniquet is applied and an IV is placed distally in the affected extremity. Exsanguination is performed with limb elevation or the assistance of an Esmarch bandage, and the cuff is inflated. Local anesthetic is then infused into the vein, which subsequently flows retrograde up the limb while diffusing into the tissues while anesthetizing neighboring nerves. ${ }^{58}$ The double tourniquet technique is used to provide anesthesia under the area of the cuff to prevent patient discomfort and procedure intolerance related to discomfort from cuff pressure. The anesthetic agent originally described by Bier was procaine, although currently newer agents such as lidocaine and ropivacaine are used more frequently, with ropivacaine possibly providing longer analgesia after cuff deflation. Adjuvants such as ketorolac, clonidine, dexmedetomidine, gabapentin, and dexamethasone have all been described in an effort to improve the quality of analgesia provided. ${ }^{56,59,60}$

\section{Special considerations for suction-assisted lipectomy}

Anesthesia techniques for liposuction remains varied based on a number of factors, including patient comorbidities, anatomical areas being treated, type of liposuction being performed, length and extent of procedure, volume of liposuction planned, and physician and patient preference. Although no evidence supports the use of any single technique, the American Society of Plastic Surgeons Practice Advisory recommends avoiding neuraxial anesthesia (ie, spinal, epidural) in office-based settings because of potential hypotension and volume overload issues. ${ }^{61}$

Over the years, the liposuction technique has evolved in an effort to minimize blood loss while maximizing removal of adipose tissue. The main variation in technique relevant to the field of anesthesia is the introduction of wet, super-wet, and tumescent techniques. In these techniques, a varying amount of solution, with or without additives, is injected into the subcutaneous space to assist with the procedure. For example, in the wet technique, the volume of injected solution is 200-300 mL. In contrast, in the tumescent technique, for each milliliter of aspirate planned, the surgeon adds $3-4 \mathrm{~mL}$ of tumescent, usually containing a local anesthetic and epinephrine. While preinfiltration practices have been proven safe procedures, such large-volume injections can lead to profound metabolic alterations as well as complications with volume overload such as pulmonary edema. Therefore, knowledge of fluid management is necessary to assist the surgeon and anesthesiologist with predicting the fluid management needs of the liposuction patient. Careful attention should be paid in all the fluids being administered during the procedure. When assessing the overall output from the procedure, it is similarly important to consider the aspirate in addition to urine and blood loss from other procedures. It is estimated that $50 \%-70 \%$ of the tumescent solution is left behind at the conclusion of the procedure. ${ }^{62}$ Of this, $70 \%$ of subcutaneous infiltrate is presumed to be intravascular. Importantly, patients with a residual volume of tumescent solution, greater than $70 \mathrm{mg} / \mathrm{kg}$, are at increased risk and need to be monitored for signs of fluid overload and will benefit from extended observation, with possible diuretic administration. ${ }^{63}$

The choice and use of anesthetics in liposuction technique has been studied. With small-volume liposuction, infiltrates with local anesthetic may be enough to provide adequate pain 
relief without the need for additional anesthesia. Marcaine may be used cautiously as an additive because of its severity of side effects, slow elimination, and inability to reverse potential toxicity. ${ }^{64}$ Lidocaine administered in wetting solutions has the potential to cause systemic toxicity. Although studies have reported administrations of up to $50 \mathrm{mg} / \mathrm{kg}$ safely, recommendations in its use include limiting the dose to $35 \mathrm{mg} / \mathrm{kg}$ and considering avoidance of the use of lidocaine when there will also be general or regional anesthesia. ${ }^{65}$ Epinephrine offers very distinct advantages including benefits to hemostasis as well as delayed absorption of the anesthetic agent; however, its use should be avoided in patients with hyperthyroidism, severe HTN, cardiac disease, and peripheral vascular disease. ${ }^{66}$

\section{Postoperative nausea and vomiting}

PONV is a common problem and can significantly impact the satisfaction or dissatisfaction with the entire surgical experience. ${ }^{67}$ For preventing PONV, the most cost-effective antiemetic combination consists of droperidol (0.625$1.25 \mathrm{mg}$ IV), dexamethasone (4-8 mg IV), and generic ondansetron (4-8 mg IV). ${ }^{68}$ Using nonnarcotic adjuvants, such as ketorolac, selective COX-2 inhibitors, and IV acetaminophen during surgery, in what is described as a "balanced" analgesic technique, is also thought to decrease PONV via its narcotic sparing potential. Unfortunately, with regard to plastic surgery, many procedures are associated with large, open surface areas, and nonsteroidal antiinflammatory drug (NSAID) usage is frequently discouraged due to its anti-platelet effect, although a recent meta-analysis of 27 studies with over 2,000 patients suggests that patients who received ketorolac intraoperatively did not experience increased adverse events or more perioperative bleeding than their controls. ${ }^{69,70}$

\section{Obstructive sleep apnea and postoperative management}

Because of the increased incidence of complications, especially respiratory, patients with OSA will typically require a greater length of close monitoring postoperatively with oxygen therapy and possibly the application of their home therapy device. According to the ASA guideline statement of 2014, patients at increased perioperative risk from OSA should not be discharged from the recovery area to an unmonitored setting until they are no longer at risk of postoperative respiratory depression. ${ }^{36}$ To establish that patients are able to maintain adequate oxygen saturation levels while breathing room air, respiratory function may be determined by observing patients in an unstimulated environment, preferably while asleep, as patients with OSA may only become hypoxemic while resting. Other recommendations include, as prior, employing narcotic-sparing analgesia methods, monitoring ventilation with capnography (ideally) or with chest wall impedance using the EKG as in most PACUs, and, if possible, keeping the patients in a semi-upright position (nonsupine if possible). Patients on continuous positive airway pressure preoperatively should resume therapy as soon as surgically feasible.

\section{Summary}

With regard to plastic surgery, the aim of ambulatory anesthesia is to provide safe and reliable anesthesia for potentially major operations while minimizing intraoperative and postoperative complications. This will allow the patient to be safely discharged and rapidly return to normal function. It is possible with the availability of rapid onset, short-acting, rapid-emergence agents with minimal postoperative side effects. To be effective however, the plastic surgeon and anesthesiologist must work together with the goal of maximizing patient safety.

\section{Disclosure}

The authors report no conflicts of interest in this work.

\section{References}

1. American Society of Plastic Surgeons. 2013 Complete Plastic Surgery Statistics Report. http://www.plasticsurgery.org/news/plastic-surgerystatistics/2013.html. Accessed August 8, 2014.

2. Michaloliakou C, Chung F, Sharma S, et al. Preoperative multimodal analgesia facilitates recovery after ambulatory laparoscopic cholecystectomy. Anesth Analg. 1996;82:44.

3. American Society of Anesthesiologists. ASA Physical Status Classification System. https://www.asahq.org/clinical/physicalstatus.htm. Accessed August 1, 2014.

4. Warner MA, Shields SE, Chute CG, et al. Major morbidity and mortality within 1 month of ambulatory surgery and anesthesia. JAMA. 1993;270:1437.

5. Fleisher LA, Beckman JA, Brown KA, et al. 2009 ACCF/AHA focused update on perioperative beta blockade incorporated into the ACC/AHA 2007 guidelines on perioperative cardiovascular evaluation and care for noncardiac surgery: a report of the American college of cardiology foundation/American heart association task force on practice guidelines. Circulation. 2009;120(21):e169.

6. Chung F, Mezei G, Tong D,et al. Adverse events in ambulatory surgery: A comparison between elderly and younger patients. Can J Anaesth. 1999;46:309.

7. Fleisher LA, Pasternak LR, Herbert R, et al. Inpatient hospital admission and death after outpatient surgery in elderly patients: Importance of patient and system characteristics and location of care. Arch Surg. 2004; 139:67-72.

8. Fisher DM. When is the ex-premature infant no longer at risk for apnea? Anesthesiology. 1995;82:807.

9. Kurth CD, LeBard SE. Association of postoperative apnea, airway obstruction, and hypoxemia in former premature infants. Anesthesiology. 1991;75:22

10. Arvidsson S. Preparation of adult patients for anaesthesia and surgery. Acta Anaesthesiol Scand. 1996;40:962. 
11. Smetana GW, Macpherson DS. The case against routine preoperative laboratory testing. Med Clin North Am. 2003;87(1):7.

12. Apfelbaum JL, Connis RT, Nickinovich DG; for Committee on Standards and Practice Parameters, Pasternak LR, Arens JF, Caplan RA, et al.; American Society of Anesthesiologists Task Force on Preanesthesia Evaluation. Practice advisory for preanesthesia evaluation: an updated report by the American Society of Anesthesiologists Task Force on Preanesthesia Evaluation. Anesthesiology. March 2012;116(3):522-538.

13. Association of Anaesthetists of Great Britain and Ireland. AAGBI safety guideline. Pre-operative assessment and patient preparation. The role of the anaesthetist. January 2010. London. http://www.aagbi.org/sites/ default/files/preop2010.pdf. Accessed August 14, 2014.

14. Archer C, Levy AR, McGregor M. Value of routine preoperative chest X-rays: a meta-analysis. Can J Anaesth. 1993;40(11):1022.

15. Lenhardt R, Seybold T, Kimberger O, et al. Local warming and insertion of peripheral venous cannulas: Single blinded prospective randomized controlled trial and single blinded randomized crossover trial. BMJ. 2002;325:409.

16. Seet E, Han TL, Chung F. Perioperative Clinical Pathways to Manage Sleep-Disordered Breathing. Sleep Med Clin. 2013;8:105.

17. Kaw R, Chung F, Pasupuleti V, Mehta J, Gay PC, Hernandez AV. Meta-analysis of the association between obstructive sleep apnoea and postoperative outcome. Br J Anaesth. 2012;109(6):897-906.

18. Weingarten TN, Flores AS, McKenzie JA, et al. Obstructive sleep apnoea and perioperative complications in bariatric patients. Br J Anaesth. 2011;106(1):131.

19. Chung F, Subramanyam R, Liao P, et al. High STOP-Bang score indicates a high probability of obstructive sleep apnoea. Br J Anaesth. May 2012;108(5):768-775.

20. Flemons WW, Whitelaw WA, Brant R, Remmers JE. Likelihood ratios for a sleep apnea clinical prediction rule. Am J Respir Crit Care Med. 1994;150(5 Pt 1):1279.

21. Netzer NC, Stoohs RA, Netzer CM, et al. Using the Berlin Questionnaire to identify patients at risk for the sleep apnea syndrome. Ann Intern Med. 1999;131(7):485.

22. Joshi GP, Ankichetty SP, Gan TJ, Chung F. Society for Ambulatory Anesthesia consensus statement on preoperative selection of adult patients with obstructive sleep apnea scheduled for ambulatory surgery. Anesth Analg. November 2012;115(5):1060-1168.

23. Grønkjær M, Eliasen M, Skov-Ettrup LS, et al. Preoperative smoking status and postoperative complications: a systematic review and metaanalysis. Ann Surg. 2014;259(1):52.

24. Wong J, Lam DP, Abrishami A, et al. Short-term preoperative smoking cessation and postoperative complications: a systematic review and meta-analysis. Can J Anaesth. 2012;59(3):268-279.

25. Yogendran S, Asokumar B, Cheng DC, et al. A prospective randomized double-blinded study of the effect of intravenous fluid therapy on adverse outcomes on outpatient surgery. Anesth Analg. 1995;80:682.

26. Mingus $\mathrm{ML}$, Bodian $\mathrm{CA}$, Bradford $\mathrm{CN}$, et al. Prolonged surgery increases the likelihood of admission of scheduled ambulatory surgery patients. J Clin Anesth. 1997;9:446.

27. Schreiner MS, Nicolson SC. Pediatric ambulatory anesthesia: NPObefore or after surgery? J Clin Anesth. 1995;7:589.

28. Maltby JR, Reid CR, Hutchinson A, et al. Gastric fluid volume and $\mathrm{pH}$ in elective inpatients: II. Coffee or orange juice with ranitidine. Can J Anaesth. 1988;35:16.

29. Eriksson LI, Sandin R. Fasting guidelines in different countries. Acta Anaesthesiol Scand. 1996;40:971.

30. Cote CJ. NPO after midnight for children: A reappraisal. Anesthesiology. 1990;72:589.

31. Goresky GV, Maltby JR. Fasting guidelines for elective surgical patients. Can J Anaesth. 1990;37:493.

32. Hutchinson A, Maltby JR, Reid CR, et al. Gastric fluid volume and $\mathrm{pH}$ in elective inpatients: I. Coffee or orange juice vs. overnight fast. Can J Anaesth. 1988;35:12.

33. Maltby JR, Pytka S, Watson NC, et al. Drinking $300 \mathrm{~mL}$ of clear fluid two hours before surgery has no effect on gastric fluid volume and $\mathrm{pH}$ in fasting and non-fasting obese patients. Can J Anaesth. 2004;51:111-115.
34. Taub PJ, Hausman L. Cosmetic Anesthesia. Plast Reconstr Surg. 2010;125(1):1e-7e.

35. Arellano R, Cruise C, Chung F, et al. Timing of the anesthetist's preoperative outpatient interview. Anesth Analg. 1989;68:645

36. American Society of Anesthesiologists Task Force on Perioperative Management of patients with obstructive sleep apnea. Practice guidelines for the perioperative management of patients with obstructive sleep apnea: an updated report by the American Society of Anesthesiologists Task Force on Perioperative Management of patients with obstructive sleep apnea. Anesthesiology. 2014;120(2):268.

37. Gan TJ, Glass PS, Windsor A, et al. Bispectral index monitoring allows faster emergence and improved recovery from propofol, alfentanil, and nitrous oxide anesthesia. Anesthesiology. 1997;87:808.

38. White PF, Ma H, Tang J, et al. Does the use of electroencephalographic bispectral index or auditory evoked potential index monitoring facilitate recovery after desflurane anesthesia in the ambulatory setting? Anesthesiology. 2004;100:811-817.

39. Rajagopalan S, Mascha E, Na J, et al. The effects of mild perioperative hypothermia on blood loss and transfusion requirement: A metaanalysis. Anesthesiology 2008;108:71-77.

40. Bremmelgaard A, Raahave D, Beir-Holgersen R, et al: Computer-aided surveillance of surgical infections and identification of risk factors. J Hosp Infect. 1989;13:1-18.

41. Staab DB, Sorensen VJ, Fath JJ, et al. Coagulation defects resulting from ambient temperature-induced hypothermia. J Trauma. 1994;36:634-638.

42. Rundgren M, Engström M. A thromboelastometric evaluation of the effects of hypothermia on the coagulation system. Anesth Analg. 2008;107(5):1465-1468.

43. Michelson AD, MacGregor H, Barnard MR, et al: Reversible inhibition of human platelet activation by hypothermia in vivo and in vitro. Thromb Haemost .1994;71:633-640.

44. Doze VA, Westphal LM, White PF, et al. Comparison of propofol with methohexital for outpatient anesthesia. Anesth Analg. 1986;65:1189.

45. Tesniere A, Servin F. Intravenous techniques in ambulatory anesthesia. Anesthesiol Clin North Am. 2003;21:273-288.

46. Erhan E, Ugur G, Anadolu O, et al. General anaesthesia or spinal anaesthesia for outpatient urological surgery. Eur J Anaesthesiol 2003;20: 647-652.

47. Pennant JH, White PF. The laryngeal mask airway: Its uses in anesthesiology. Anesthesiology. 1993;79:144.

48. Sa Régo M, Watcha MF, White PF, et al. The changing role of monitored anesthesia care in the ambulatory setting. Anesth Analg. 1997;85:1020-1036

49. Song D, Greilich NB, White PF, et al. Recovery profiles and costs of anesthesia for outpatient unilateral inguinal herniorrhaphy. Anesth Analg. 2000;91:876-881.

50. Price R. Transient femoral nerve palsy complicating preoperative ilioinguinal nerve blockade for inguinal herniorrhaphy. Br J Surg. $1995 ; 82: 137$.

51. Zide BM, Swift R. How to block and tackle the face. Plast Reconstr Surg. 1998;101(3):840-851.

52. Strazar R, Leynes PG, Lalonde DH. Minimizing the pain of local anesthesia injection. Plast Reconstr Surg. 2013;132(3):675-684.

53. Hadj A, Rosenfeldt F, Nicholson D, et al. Safety and efficacy of extended-release bupivacaine local anaesthetic in open hernia repair: a randomized controlled trial. ANZ J Surg. 2012;82(4):251-257.

54. Morales R Jr, Mentz H, Newall G, Patronella C, Masters O. Use of abdominal field block injections with liposomal bupivicaine to control postoperative pain after abdominoplasty. Aesthet Surg J. 2013;33(8):1148-1153.

55. Urmey WF, Stanton J, Peterson M, et al. Combined spinal-epidural anesthesia for outpatient surgery: Dose-response characteristics of intrathecal isobaric lidocaine using a 27-gauge Whitacre spinal needle. Anesthesiology. 1995;83:528.

56. Joshi GP. Combined spinal/epidural anesthesia for outpatient surgery. Anesthesiology. 1996;84:481. 
57. Bou-Merhi JS, Gagnon AR, St Laurent JY, Bissonnette M, Chollet AA. Intravenous regional anesthesia administered by the operating plastic surgeon: is it safe and efficient? Experience of a medical center. Plast. Reconstr. Surg. 2007;120(6);1591-1597.

58. Atanassoff PG, Ocampo CA, Bande MC, et al. Ropivacaine $0.2 \%$ and lidocaine $0.5 \%$ for intravenous regional anesthesia in outpatient surgery. Anesthesiology. 2001;95:627-631.

59. Viscomi CM, Friend A, Parker C, et al. Ketamine as an adjuvant in lidocaine intravenous regional anesthesia: A randomized, double-blind, systemic control trial. Reg Anesth Pain Med. 2009;34:130-133.

60. Tryba M, Gehling M. Clonidine: A potent analgesic adjuvant. Curr Opin Anaesthesiol. 2002;15:511-517.

61. Iverson RE, Lynch DJ. American Society of Plastic Surgeons Committee on Patient Safety. Practice advisory on liposuction. Plast Reconstr Surg. 2004;113:1478-1490; discussion 1491.

62. Trott SA, Beran SJ, Rohrich, et al. Safety considerations and fluid resuscitation in liposuction: An analysis of 53 consecutive patients. Plast. Reconstr. Surg. 1998;102:2220.

63. Gingrass M. Lipoplasty complications and their prevention. Clin. Plast. Surg. 1999;26:341.
64. Miller RD. Anesthesia, 5th Ed. Philadelphia: Churchill Livingstone, 2000;503-517.

65. Perry, A. W., Petti, C., and Rankin, M. Lidocaine is not necessary in liposuction. Plast. Reconstr. Surg. 1999;104:1900.

66. Matarasso, A. The tumescent technique: The effect of high tissue pressure and dilute epinephrine on absorption of lidocaine. Plast. Reconstr. Surg. 1999;103:997.

67. Eberhart LH, Morin AM, Wulf H, et al. Patient preferences for immediate postoperative recovery. Br J Anaesth. 2002;89:760.

68. Glass PS, White PF. Practice guidelines for the management of postoperative nausea and vomiting: past, present, and future. Anesth Analg. 2007; 105:1528-1529.

69. Souter AJ, Fredman B, White PF, et al. Controversies in the perioperative use of nonsteroidal antiinflammatory drugs. Anesth Analg. 1994;79:1178.

70. Gobble RM, Hoang HL, Kachniarz B, Orgill DP. Ketorolac does not increase perioperative bleeding: a meta-analysis of randomized controlled trials. Plast Reconstr Surg. 2014;133(3):741-755.
Ambulatory Anesthesia

\section{Publish your work in this journal}

Ambulatory Anesthesia is an international, peer reviewed, open access journal publishing articles that address all aspects of ambulatory anesthesia practice, in particular: anesthetic techniques, sedation and safety practices, pharmacokinetics, preoperative evaluation, analgesia interventions, regulatory and compliance issues, postoperative recovery,

\section{Dovepress}

patient satisfaction, administrative topics, and cost analysis themes. The manuscript management system is completely online and includes a very quick and fair peer review system, which is all easy to use. Visit $\mathrm{http}: / /$ www.dovepress.com/testimonials.php to read real quotes from published authors. 